\title{
Progressive CT findings and positive RT-PCR again of recovered and discharged patients with COVID-19
}

\author{
Lu Huang ${ }^{1 \#}$, He Deng ${ }^{2 \#}$, Liming Xia ${ }^{1}$, Xin Zhou ${ }^{2}$ \\ ${ }^{1}$ Department of Radiology, Tongji Hospital, Tongji Medical College, Huazhong University of Science and Technology, Wuhan, China; ${ }^{2}$ Innovation \\ Academy for Precision Measurement Science and Technology, Chinese Academy of Sciences, Wuhan, China \\ \#These authors contributed equally to the work. \\ Correspondence to: Liming Xia. Department of Radiology, Tongji Hospital, Tongji Medical College, Huazhong University of Science and Technology, \\ Wuhan 430030, China. Email: Imxia@tjh.tjmu.edu.cn; Xin Zhou. Innovation Academy for Precision Measurement Science and Technology, Chinese \\ Academy of Sciences, Wuhan 430071, China. Email: xinzhou@wipm.ac.cn. \\ Provenance and Peer Review: This article was a free submission to the editorial office, Fournal of Thoracic Disease. The article was sent for external peer \\ review by the editorial office.
}

Submitted Mar 21, 2020. Accepted for publication May 22, 2020.

doi: $10.21037 /$ jtd-20-1417

View this article at: http://dx.doi.org/10.21037/jtd-20-1417

As of April 27, 2020, 2,878,196 (85,530 new) COVID-19 patients were confirmed across the world (1), within which 77,555 (82,836 confirmed) recovered in China. Previous studies focused on clinical and radiological features of patients with confirmed infection $(2,3)$, or recovered patients with no symptoms and no changes of follow-up chest computed tomography (CT) findings (4). To our knowledge, both the progressive CT findings and fever again of recovered and discharged patients has not been reported.

One representative hospitalized patient with COVID-19 treated at Tongji Hospital, Wuhan, China, from January 20, 2020, to February 8, 2020 was discharged and isolated at home, because he met the discharge criteria in China: (I) normal temperature for more than 3 days; (II) resolved respiratory symptoms; (III) substantially improved acute exudative lesions on CT images; and (IV) 2 consecutively negative reverse transcription polymerase chain reaction (RT-PCR) test results separated by at least 24 hours (5). However, on February 13, 2020, the patient was admitted to the isolation ward and received supplemental oxygen through nasal cannula once again, owing to symptoms of fever and shortness of breath, and progressive CT findings. RT-PCR test was performed via nasopharyngeal swabs, and severe acute respiratory syndrome coronavirus 2 (SARSCoV-2) nucleic acid was positive on February 14, 2020. The RT-PCR test performed in Tongii Hospital was reasonably reliable, due to the RT-PCR detection reagent used in the clinical laboratory proved by China Food and Drug Administration (CFDA) with $100 \%$ positive and negative reference coincidence rate.

Laboratory studies, clinical data, and radiological features were collected from electronic medical records. The local ethical review board approved this study and waived the requirement to obtain individual informed consent.

A 40-year-old patient (male) living in Wuhan, was admitted to a fever clinic on January 20, 2020, with symptoms of fever for 2 days. Chest CT revealed multiple ground-glass opacities (GGOs) distributed in the peripheral and peribronchovascular area of bilateral lungs (Figure 1A). The major axis of maximal opacity was $2.8 \mathrm{~cm}$ in right lower lobe. Respiratory tract virus antigen and antibody tests, including cytomegalovirus, influenza virus A and B, human parainfluenza virus, respiratory syncytial virus and adenovirus, showed all negative results on January 22, 2020, and SARS-CoV-2 nucleic acid was positive one day later. A 4-day follow-up CT showed multiple GGOs enlarged significantly with multifocal consolidation, interlobular septal thickening, vessel thickening and air bronchogram, which involved all pulmonary segments (Figure 1B). The major axis of maximal lesion was $7.5 \mathrm{~cm}$ in right lower lobe. On January 27, 2020, the 2nd follow-up CT illustrated multiple GGOs further enlarged (the major axis of maximal lesion was $10.1 \mathrm{~cm}$ in right lower lobe), but consolidation 


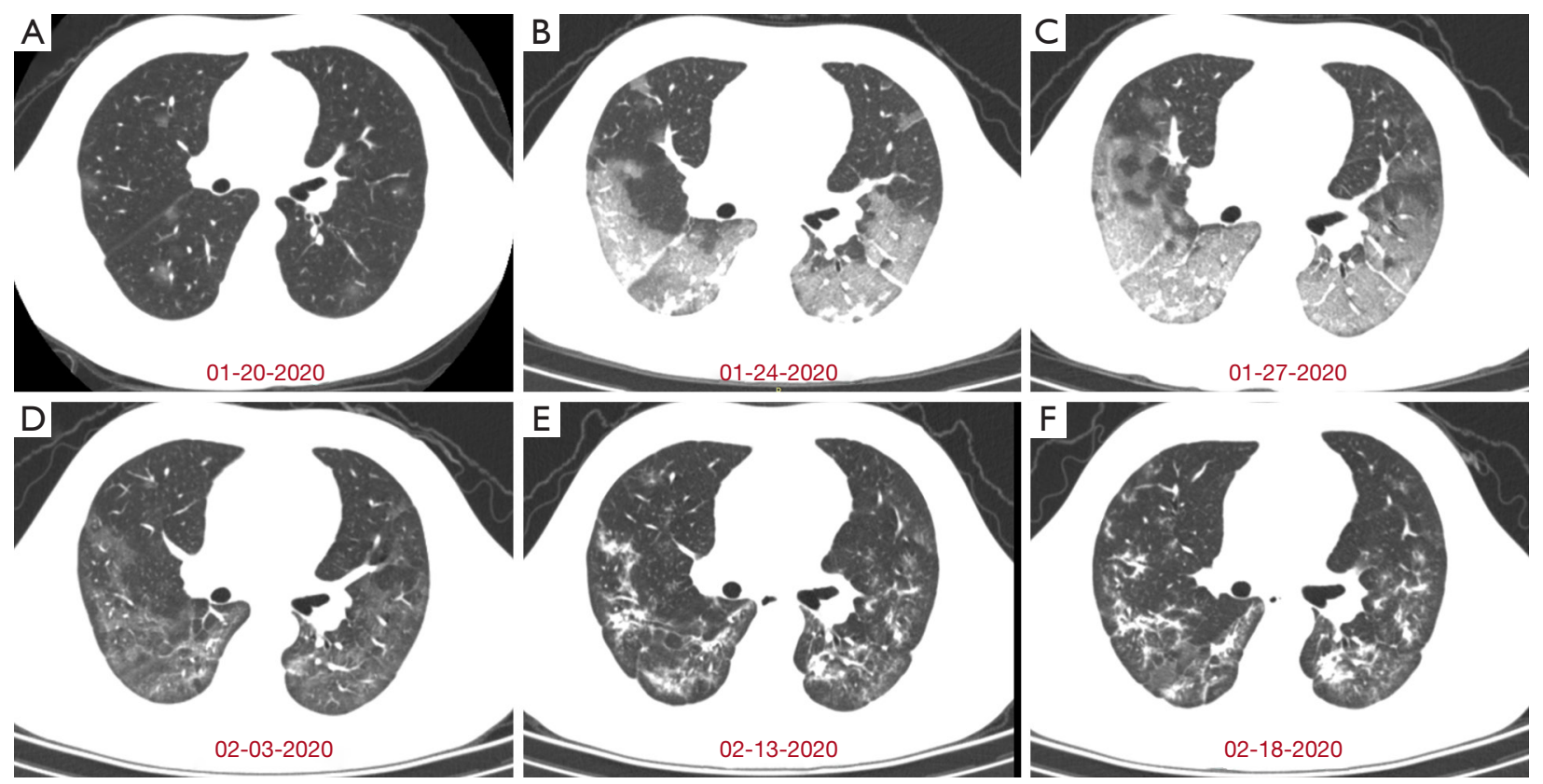

Figure 1 Series of chest CT images in the recovered and discharged patient with symptoms of fever and shortness of breath again. (A) On January 20, 2020, initial scan for the first hospital admission showed multiple ground-glass opacities (GGOs) in bilateral lungs. (B) A 4-day follow-up scan showed multiple GGOs enlarged with multifocal consolidation, interlobular septal thickening, and vessel thickening. (C) The follow-up CT 3 days later showed GGOs enlarged significantly with interlobular thickening, but some consolidation opacities absorbed. (D) The follow-up CT 7 days later showed the most GGOs absorbed. (E) On February 13, 2020, initial scan for the second hospital admission showed progression from some GGOs to multifocal consolidation. (F) A 5-day follow-up scan showed consolidation opacity enlarged in left lower lobe.

partial absorbed (Figure 1C). After 7 days, the 3rd follow-up CT represented remarkable absorption of multiple GGOs in bilateral lungs and multiple linear consolidation (Figure 1D). The patient remained negative after 2 consecutive RT-PCR tests on February 4 and 6, 2020. The time from symptom onset to recovery was 22 days.

After hospital discharge, the patient was isolated at home for 5 days. On day 27 of illness, the patient had a fever again $\left(38.2{ }^{\circ} \mathrm{C}\right)$, and $\mathrm{CT}$ showed some GGOs absorbed but others progressed to multifocal organizing consolidation in bilateral lungs (Figure 1E). Positive RT-PCR test results and laboratory studies suggested inflammatory situation, with lymphocyte count 0.70 (normal: $1.10-3.20) \times 10^{9} / \mathrm{L}$, erythrocyte sedimentation rate 68 (normal: $0-15) \mathrm{mm} / \mathrm{H}$, procalcitonin 0.15 (normal: $0.02-0.05) \mathrm{ng} / \mathrm{mL}$, and lactate dehydrogenase (LDH) 293 (normal: 135-225) U/L.

On February 18, 2020, the follow-up CT showed some consolidation enlarged in left lower lobe and right upper lobe, while other linear consolidation remained (Figure $1 F$ ). Moreover, laboratory studies matched the progressive
CT findings, with ferritin 1,846.5 (normal: 30-400) $\mu \mathrm{g} / \mathrm{L}$, procalcitonin 0.13 (normal: $0.02-0.05$ ) $\mathrm{ng} / \mathrm{mL}$, interleukin-2 receptor 1,108 (normal: 223-710) U/mL, interleukin-6 9.97 (normal: <7) pg/mL, interleukin-10 10.2 (normal: <9.1) pg/mL, and tumor necrosis factor 15.7 (normal: <8.1) pg/mL. From February 13, 2020, the patient was admitted to the isolation ward and received antibiotic, antiviral, hormone, and stomach-protecting therapies once again.

On February 29, 2020 , the LDH (162 U/L), procalcitonin $(0.03 \mathrm{ng} / \mathrm{mL})$, and lymphocyte count $\left(1.28 \times 10^{9} / \mathrm{L}\right)$ recovered, RT-PCR tests were negative, and CT scan showed multiple consolidative opacities were partially absorbed in bilateral lungs. Two days later, the serological SARS-CoV-2 antibody test was positive with IgM 19.27 (normal: $\leq 10$ ) AU/mL and SARS-CoV-2 IgG 24.68 (normal: $\leq 10$ ) AU/mL, and ferritin $630.6 \mu \mathrm{g} / \mathrm{L}$ elevated significantly. The patient was still in the hospital for treatment because of high antibodies and ferritin.

A patient with COVID-19 who satisfied the criteria for discharge (absence of clinical symptoms, substantial 
improvement of acute exudative lesion on CT images, and 2 consecutively negative RT-PCR test results) (5) had a fever, processive CT findings and positive RT-PCR test results 5 days later. These findings indicated that at least a proportion of recovered patients might still carry virus, though his SARS-CoV-2 nucleic acid tests were negative through twice nasopharyngeal swabs. We speculated that SARS-CoV-2 was still active in lower respiratory tract, which was also supported by multiple GGOs and linear consolidation on CT images.

Noticeably, 3 days before discharge, the procalcitonin $(0.04 \mathrm{ng} / \mathrm{mL})$ and lymphocyte count $\left(1.27 \times 10^{9} / \mathrm{L}\right)$ recovered but LDH (313 U/L) was still significantly high. This suggested that the pneumonia had not been completely absorbed. On February 28, the press conference of the joint prevention and control mechanism of the state council in China, reported a few discharged cases with positive RTPCR tests from different provinces. It indicated that current criteria for hospital discharge could be reevaluated.

Our finding suggested that current discharge criteria could take into account some laboratory studies, such as interleukin6, LDH, and SARS-CoV-2 IgM and IgG. In the future, we will investigate longitudinal studies on a large cohort, which can help understand chest morphometry of COVID-19 and provide a useful path towards improvement of clinical strategies against the disease.

\section{Acknowledgments}

Funding: This work was supported by National Natural Science Foundation of China $(81625011,81771917$, 91859206), National Key R\&D Program of China (2018YFA0704000), Key Research Program of Frontier Sciences, CAS (ZDBS-LY-JSC004).

\section{Footnote}

Conflicts of Interest: All authors have completed the ICMJE uniform disclosure form (available at http://dx.doi. org/10.21037/jtd-20-1417). The authors have no conflicts of interest to declare.

Ethical Statement: The authors are accountable for all aspects of the work in ensuring that questions related to the accuracy or integrity of any part of the work are appropriately investigated and resolved.

Open Access Statement: This is an Open Access article distributed in accordance with the Creative Commons Attribution-NonCommercial-NoDerivs 4.0 International License (CC BY-NC-ND 4.0), which permits the noncommercial replication and distribution of the article with the strict proviso that no changes or edits are made and the original work is properly cited (including links to both the formal publication through the relevant DOI and the license). See: https://creativecommons.org/licenses/by-nc-nd/4.0/.

\section{References}

1. Coronavirus disease 2019 (COVID-19) Situation Report-98. Available online: https://www.who.int/ emergencies/diseases/novel-coronavirus-2019/situationreports. Accessed 27 Apr 2020.

2. Huang C, Wang Y, Li X, et al. Clinical features of patients infected with 2019 novel coronavirus in Wuhan, China. Lancet 2020;395:497-506.

3. Wu F, Zhao S, Yu B, et al. A new coronavirus associated with human respiratory disease in China. Nature 2020;579:265-9.

4. Lan L, Xu D, Ye G, et al. Positive RT-PCR Test Results in Patients Recovered From COVID-19. JAMA 2020;323:1502-3.

5. China National Health Commission. Diagnosis and treatment of 2019-nCoV pneumonia in China. In Chinese. Available online: http://www.nhc.gov.cn/yzygj/s7653p/202 003/46c9294a7dfe4cef80dc7f5912eb1989.shtml. Accessed 4 Mar 2020.
Cite this article as: Huang L, Deng H, Xia L, Zhou X. Progressive CT findings and positive RT-PCR again of recovered and discharged patients with COVID-19. J Thorac Dis 2020;12(6):3439-3441. doi: 10.21037/jtd-20-1417 